ARTICLE

\title{
Mechanistic insight into copper cation exchange in cadmium selenide semiconductor nanocrystals using X-ray absorption spectroscopy
}

\author{
Alex Khammang ${ }^{1}$, Joshua T. Wright ${ }^{2} \&$ Robert W. Meulenberg (iD ${ }^{1 凶}$
}

In terms of producing new advances in sustainable nanomaterials, cation exchange (CE) of post-processed colloidal nanocrystals (NCs) has opened new avenues towards producing non-toxic energy materials via simple chemical techniques. The main processes governing $\mathrm{CE}$ can be explained by considering hard/soft acid/base theory, but the detailed mechanism of $\mathrm{CE}$, however, has been debated and has been attributed to both diffusion and vacancy processes. In this work, we have performed in situ x-ray absorption spectroscopy to further understand the mechanism of the CE of copper in solution phase CdSe NCs. The x-ray data indicates clear isosbestic points, suggestive of cooperative behavior as previously observed via optical spectroscopy. Examination of the extended x-ray absorption fine structure data points to the observation of interstitial impurities during the initial stages of $C E$, suggesting the diffusion process is the fundamental mechanism of CE in this system.

\footnotetext{
${ }^{1}$ Department of Physics and Astronomy and Frontier Institute for Research in Sensor Technologies, University of Maine, Orono, ME 04469, USA.

${ }^{2}$ Department of Physics, Illinois Institute of Technology, Chicago, IL, USA. ${ }^{凶}$ email: robert.meulenberg@maine.edu
} 
S ince the first synthesis of high-quality nanocrystals (NCs) by hot-injection methods ${ }^{1}$, many research groups have sought to find comparable syntheses for a wide range of inorganic materials. Although a host of materials systems have been successfully synthesized ${ }^{2-5}$, the controllable synthesis of other systems, such as the Group IV NC system, has been difficult. The inability to make just about any binary NC system, however, was solved using the cation exchange (CE) approach. The first report of CE in NCs reported the fast transformation ( $<1 \mathrm{~min})$ of CdSe NCs into $\mathrm{Ag}_{2} \mathrm{Se}$ by introducing a methanolic $\mathrm{Au}^{+}$solution into a colloidal suspension of CdSe $\mathrm{NCs}^{6}$. The primary driving force (energetically) behind $\mathrm{CE}$ processes can be explained by considering hard/soft acid/base (HSAB) theory, although there are a host of parameters than can influence this process. Initially suggested by the Alivisatos group ${ }^{6}$, this model implies that the "hardness" or "softness" of the materials undergoing $\mathrm{CE}$ will drive the exchange process. For example, if we consider the well-known example of $\mathrm{Cu}^{+}$cation exchange in CdSe NCs ${ }^{7,8}$ in the core NC we have a soft acid (cadmium cation, $\mathrm{Cd}^{2+}$ ) and a soft base (selenium anion, $\mathrm{Se}^{2-}$ ) with the incoming cation a soft acid (copper cation, $\mathrm{Cu}^{+}$). As the $\mathrm{Cu}^{+}$ion is softer than the $\mathrm{Cd}^{2+}$ ion, with an appropriate hard base (such as methanol, $\mathrm{MeOH}$ ), the $\mathrm{CE}$ process can be favored via a simple soft acid/soft base combination, $\mathrm{Cu}^{+} / \mathrm{Se}^{2-}$.

Since the early reports of complete structural conversion via $\mathrm{CE}$, researchers have shown that partial exchange of the NC lattice is possible, but the models behind the partial exchange process are debated. One model (that we will call the interstitial model) suggests that partial cation exchange in a $A B$ lattice (where $\mathrm{A}$ is a cation and $\mathrm{B}$ is an anion) is governed by a multistep process that begins with the diffusion of the impurity ion $\left(\mathrm{M}^{\mathrm{n}+}\right)$ into $\mathrm{NC}$ with the impurity ion sitting on an interstitial site $^{9}$. If more than one impurity ion is introduced to the NC, the strong Coulomb interaction between the two $\mathrm{M}^{\mathrm{n}+}$ force the ions to the surface. Following surface segregation, the $\mathrm{M}^{\mathrm{n}+}$ can substitute for a host cation via a kick-off reaction, where $\mathrm{M}^{+}$substitutes for the host cation site (substitutionally) leading to an $\mathrm{M}^{-}$ site. This new negatively changed site can accumulate the interstitial $\mathrm{M}^{\mathrm{n}+}$ ions leading to local pockets of "MB" material. This process can repeat until the formation of the $\mathrm{M}_{2} \mathrm{~B}$ material. Another model (that we will call the vacancy model) for the CE process in NCs is a vacancy driven formation ${ }^{10}$. In this case, the NC must be imperfect, meaning it must possess surface vacancies. With the existing surface vacancy, a host cation transfers to the vacant surface site, and a surface $\mathrm{M}^{\mathrm{n}+}$ ion hops to the vacancy left behind by the host cation. The host cation, now on the surface, desorbs as a metal complex and a "new" $\mathrm{M}^{\mathrm{n}+}$ ion adsorbs onto the NC surface. This process continues to completion. In addition, it has been recently suggested that CE in CdSe (with Ag or $\mathrm{Cu}$ ) exhibits positive cooperativity, meaning that only two phases are present during the CE process ${ }^{7}$.

While there have been a large number of studies that investigate the possible mechanism for $\mathrm{CE}$ in NCs, there still exists some debate on the exact mechanism as the process seems to be highly dependent on a number of factors including crystal type, particle shape, and surface ligand type ${ }^{8-13}$. For cation exchange of $\mathrm{Cu}$ in CdSe NCs, it has been generally suggested that the process may proceed via an interstitial mechanism, but there has been no direct evidence of this. In this work, we investigate the mechanism of cation exchange in CdSe NCs using in situ x-ray absorption spectroscopy (XAS). The use of operando or in situ XAS for probing catalytic materials has been well documented ${ }^{14-17}$, but progress in applying these techniques related to studying the complex chemistry and physics of colloidal nanostructures has been slower. One of the earliest uses of in situ XAS was by the Alivisatos group ${ }^{6}$ which showed that $\mathrm{Ag}^{+}$cation exchange in
CdSe NCs can be described as a rapid transformation between two species, CdSe and $\mathrm{Ag}_{2} \mathrm{Se}$, with no contributions from impurity phases, yet no detailed mechanism for the process was ascertained. Kompch and co-workers have performed the most detailed XAS work on CE in $\mathrm{CdSe}^{18}$ where the mechanism of $\mathrm{Ag}$ $\mathrm{CE}$ in CdSe NC was probed via reverse Monte Carlo analysis of the extended $\mathrm{x}$-ray absorption fine structure (EXAFS) data, although this study focused on solid-phase materials. Inspired by this work and the work of the Jain group ${ }^{7}$, in our current work, we use partial cation exchange of $\mathrm{Cu}$ to probe various stages of the CE reaction in solution phase CdSe NCs. Analysis of the EXAFS data show evidence for interstitials during the CE process. In addition, our XAS results suggest the CE process exhibits positive cooperativity, in agreement with prior results 7 . Our measurements are the first solution based measurements that provide structural evidence for interstitial inclusions and show that in situ XAS is a powerful and local probe to investigate chemical mechanisms in NCs.

\section{Results}

Copper cation exchange in CdSe NCs as probed by X-ray absorption spectroscopy. The CdSe NCs were synthesized through a colloidal hot-injection method as previously reported ${ }^{19,20}$ (see Methods for details). These synthetic methods can produce wurtzite CdSe NCs with highly tunable sizes; in this work, we have studied three distinct (nominal) sizes of NCs: 4.0, 5.0 , and $6.0 \mathrm{~nm}$ diameter. We also attempted measurements on $3.0 \mathrm{~nm}$ CdSe NCs. Due to both air and beam instability, highquality EXAFS data was not attainable. But $\mathrm{x}$-ray absorption near edge structure (XANES) data was obtained and shows the same general trends as the other particle sizes (see Fig. S4). The cation exchange reaction is initiated by adding an appropriate molar fraction of the metal salt to the QD solution ${ }^{7}$. Because the reaction is reactant limited, a sub-stoichiometric amount of the metal cation salt (in this work we used tetrakis(acetonitrile)copper(I) hexafluorophosphate, $\mathrm{Cu}(\mathrm{I}) \mathrm{PF}_{6}$; see Methods section) can be added offering the ability to probe various stages of the chemical reaction. The exact details of the cation salt loading are shown in Supplementary Table S1. As the ethanolic copper salt solution is added to the NC solution, $\mathrm{x}$-ray absorption fine structure (XAFS) measurements were conducted at the $\mathrm{Cd}, \mathrm{Se}$, and $\mathrm{Cu}$ K-edges. We also attempted some XAFS measurements of Ag cation exchange in CdSe. These results, while preliminary, are qualitatively similar to our observations for $\mathrm{Cu}$ cation exchange (Figures S12 and S13). It should be noted that the kinetics of this reaction (less than one second) are too fast to monitor via traditional XAFS setups without using microfluidic reactors ${ }^{21}$. Attempts to use metal salts that slowed the cation exchange reaction ${ }^{22}$ to allow kinetic XAFS studies were unsuccessful.

In Fig. 1, representative XANES spectra are displayed for 5.0 $\mathrm{nm}$ CdSe NCs. XANES spectra for other sizes can be found in the Supplementary Information. At the lowest $\mathrm{Cu}$ salt loading levels $(\mathrm{mol} \mathrm{Cu} / \mathrm{mol} \mathrm{Cd}=0.01 ; \sim 12 \mathrm{Cu}$ atoms/NC), no distinct changes in the $\mathrm{Cd}$ or Se K-edge XANES features are evident, and there is no detectable signal for the $\mathrm{Cu} \mathrm{K}$-edge. This is not totally unexpected, as the equivalent amount of $\mathrm{Cu}$ present in the interrogation volume of the XAFS experiment is $\sim 2 \mathrm{nmol}$. At the second concentration step $(\mathrm{mol} \mathrm{Cu} / \mathrm{mol} \mathrm{Cd}=0.04 ; \sim 47 \mathrm{Cu}$ atoms/NC), we observe a very weak $\mathrm{Cu} \mathrm{K}$-edge signal, with no strong changes in the $\mathrm{Cd}$ or Se K-edges (SI Figure S5). It is noteworthy that the $\mathrm{Cu}$ K-edge spectrum for $\sim 47 \mathrm{Cu}$ ions/NC is distinct from the spectrum for $\mathrm{Cu}(\mathrm{I}) \mathrm{PF}_{6}$ (Fig. 1c,vii) suggesting incorporation of $\mathrm{Cu}$ into the CdSe NC. As the amount of $\mathrm{Cu}$ in the CdSe solution is continually increased, subsequent changes in the XANES features are evident. Both the Cd and Se K-edges 

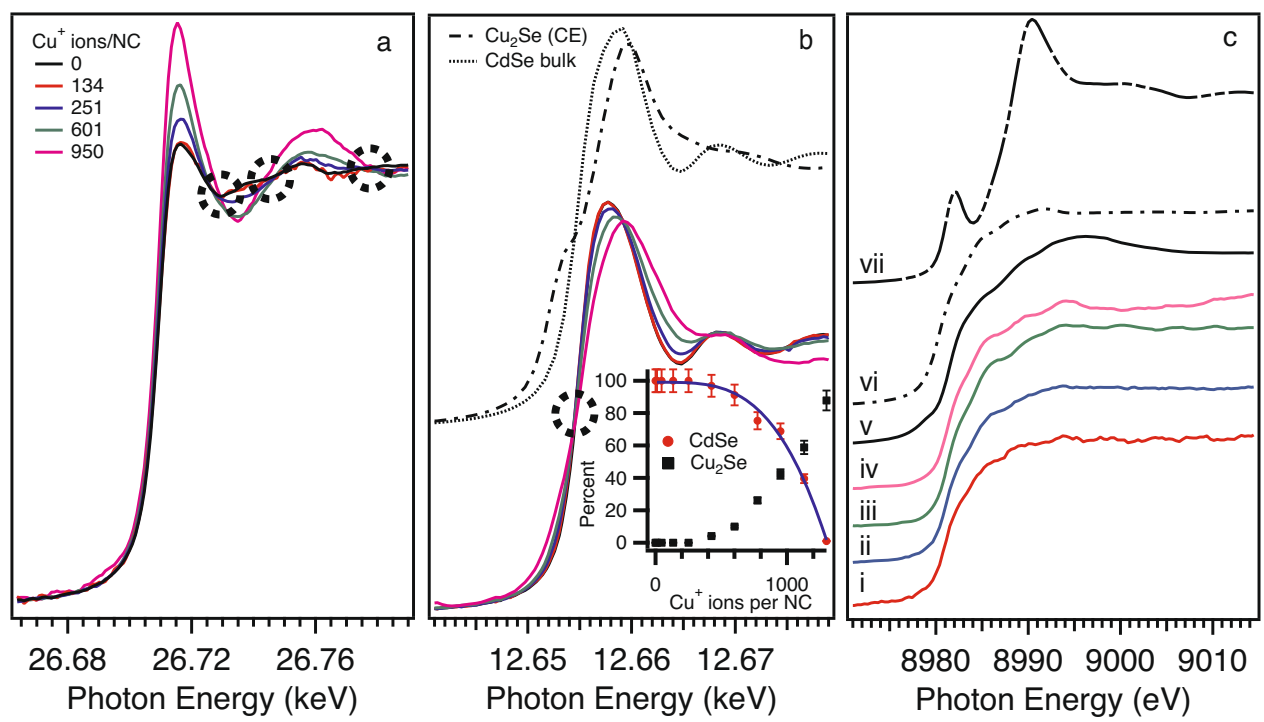

Fig. 1 Core level XANES during the cation exchange reaction. X-ray absorption spectra for $5.0 \mathrm{~nm}$ CdSe NCs at the (a) Cd K-edge, (b) Se K-edge, and (c) Cu K-edge for (i) 134, (ii) 251, (iii) 601, (iv) $950 \mathrm{Cu}$ ions/NC, (v) $\mathrm{Cu}_{2} \mathrm{Se}$ via cation exchange of CdSe, (vi) commercial $\mathrm{Cu}_{2} \mathrm{Se}_{\text {, and }}$ (vii) $\mathrm{Cu}_{\text {(I)PF }}$. The inset of (b) are the LCF results for the Se K-edge using a CdSe and $\mathrm{Cu}_{2} \mathrm{Se}$ model. The error bars in the inset represent uncertainties in the LCF.

show gradual changes in the XANES with increasing copper concentration with clear existence of isosbestic points (dotted circles in Fig. 1a and b). As increasing amounts of $\mathrm{Cu}$ are added to the CdSe solution, transformation of the CdSe species to $\mathrm{Cu}_{2} \mathrm{Se}$ is evident via Se K-edge XANES (Fig. 1b). Principal component analysis of the Se K-edge XANES suggests only two species are present (consistent with the isosbestic points) and linear combination fitting (LCF) indicates complete transformation of the $\mathrm{CdSe} \rightarrow \mathrm{Cu}_{2} \mathrm{Se}$ for $\mathrm{Cu}$ mol\% $>50 \%$. Below a concentration of $\sim 600 \mathrm{Cu}$ ions/NC, there is a very small fraction of $\mathrm{Cu}_{2} \mathrm{Se}$ which is similar to observations by the Jain group who used optical spectroscopy to probe the $\mathrm{CE}$ reaction ${ }^{7}$. The CdSe fraction as a function of $\mathrm{Cu}$ concentration can be fit to the Hill equation (solid line in the inset of Fig. 1b) with a Hill coefficient of $n=3.5$, which is consistent with positive cooperativity and the results from the Jain group. Meanwhile, with increasing $\mathrm{Cu}$ concentration the $\mathrm{Cd}$ K-edge XANES exhibits a transformation from CdSe to a five or six-fold coordinated $\mathrm{Cd}$ species (vide infra). This behavior is strongly suggestive of cooperative behavior consistent with the LCF and again supports conclusions by the Jain group ${ }^{7}$.

Cation exchange inclusion models: Vacancy mediated versus interstitial inclusions. When considering possible mechanisms for $\mathrm{CE}$ in NCs, the two most common candidates are diffusion and vacancy models. For low $\mathrm{Cu}$ concentrations (i.e., partial $\mathrm{CE}$ ), we would expect a significant fraction of $\mathrm{Cu}$ atoms in interstitial lattice sites for a pure diffusion model, while pre-existing vacancies can allow for direct substitutional incorporation of the $\mathrm{Cu}$ dopant. To ascertain the plausibility of the two models, we constructed cluster models of CdSe (125 atoms) and consider four cases: (a) undoped CdSe, (b) a substitutional $\mathrm{Cu}$ dopant, (c) an interstitial $\mathrm{Cu}$ dopant, and (d) two interstitial $\mathrm{Cu}$ dopants. (Figure 2a). We then computed the theoretical EXAFS spectra for the Cd K-edge using FEFF9 ${ }^{23}$. In the FEFF calculations, for selfconsistency, we used a $5 \AA$ cluster and considered the effects of a core hole using the final state rule. Figure $2 c$ displays the Fourier transformed (FT) EXAFS magnitude for the various cluster models. We note that the FEFF calculation allows the extraction of EXAFS data out to $k=20 \AA^{-1}$, and while good experimental EXAFS data can extend to $k=15 \AA^{-1}$, in the case of NC samples (i.e., like we are studying) $k=10-12 \AA^{-1}$ is typical. We therefore
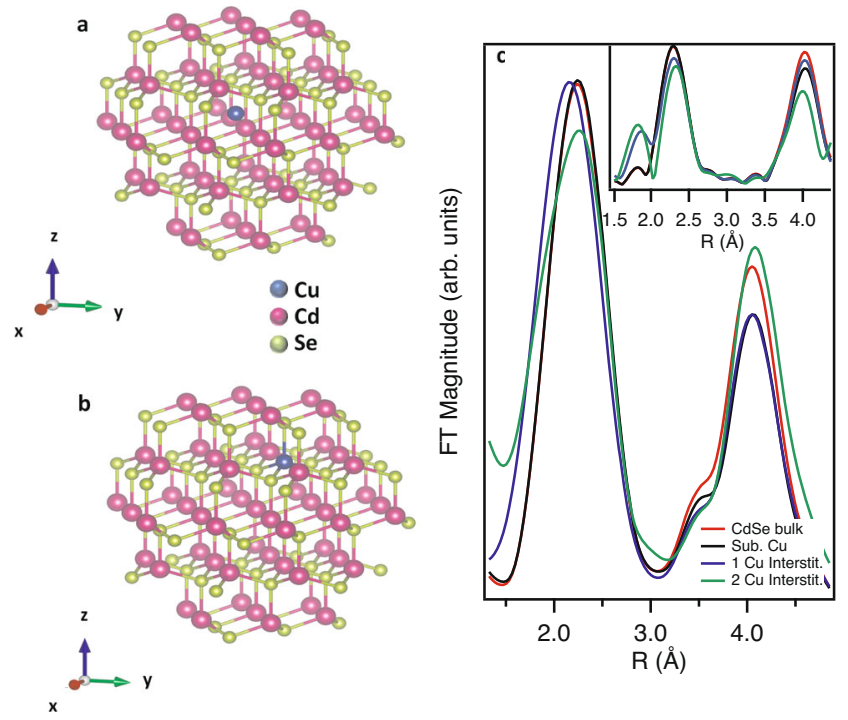

Fig. 2 Structural models of $\mathrm{Cu}$ atom inclusion in $\mathrm{CdSe}$ and theoretical Fourier transformed EXAFS data. CdSe cluster model showing (a) substitutional and (b) interstitial Cu dopants and (c) theoretical Fourier transformed EXAFS magnitude for the Cd K-edge for CdSe bulk and substitutional and interstitial Cu dopants. See text for differences between (c) and the inset for (c).

display the theoretical FT EXAFS data with a transform range $3 \leq k \leq 15 \AA^{-1}$ in the inset of Fig. 2 c. For a substitutional $\mathrm{Cu}$ impurity, very little deviation from the undoped spectrum is observed. This is not surprising, as the main scattering peak is related to the nearest neighbors $(\mathrm{NN})$, and the $\mathrm{Cu}$ substitutional impurity sits in a next-nearest neighbor (NNN) lattice position. This explains why there is a change to the scattering peak at $\sim 4.0 \AA$ as that peak is related to NNN contributions. Realistically, however, we cannot access these features for our NC samples, so we will focus solely on analysis of the main scattering peak (NN contributions). For interstitial impurities, of particular note is the appearance of scattering features at values of $R \sim 1.9 \AA$ (not phase shift corrected). Since a FT window of $3 \leq k \leq 15 \AA^{-1}$ is not experimentally feasible in our system, we also plot the theoretical 
FT EXAFS data for a FT window of $3 \leq k \leq 10 \AA^{-1}$ (Fig. 2c), which should allow a more direct comparison to our experimental data. Of note is the smearing of the low $R$ scattering feature into the main peak which results in a single peak that is shifted slightly to lower $R$ (by $\sim 0.06 \AA$ ) with a broadening of the main peak $(\sim 10 \%)$ due to the appearance of interstitial $\mathrm{Cu}$. As more $\mathrm{Cu}$ is introduced into the model, an increased broadening of the main peak is observed. We hypothesize, therefore, that visual inspection for broadening of the main scattering peak in the $\mathrm{Cd}$ K-edge FT EXAFS data should allow for the identification of interstitial $\mathrm{Cu}$ impurities.

Evidence for interstitials during cation exchange. Experimental Cd K-edge FT EXAFS data is presented in Fig. 3a for $5.0 \mathrm{~nm} \mathrm{CdSe}$ $\mathrm{NCs}$ at various stages of the cation exchange reaction. Some striking behavior is immediately observable by the eye. First, the broadening of the NN scattering peak increases with increasing $\mathrm{Cu}$ salt addition. Second, a shift to lower $R$ is evident with increasing $\mathrm{Cu}$ salt content. Based upon our FEFF simulations, these observations strongly suggest the appearance of interstitial $\mathrm{Cu}$ atoms during the $\mathrm{CE}$ reaction in CdSe NCs. If the CE reaction was proceeding via a purely substitutional process, we would not expect any broadening or shifting of the NN peak; indeed, a decrease in peak amplitude would be the expectation. We would like to point out that this broadening is not a consequence of a contribution of components of $\mathrm{Cd}$ bonded to low- $Z$ elements (i.e., $\mathrm{Cd}-\mathrm{O})$. We assert this for two reasons: (1) the $\mathrm{Cd}-\mathrm{O}$ complexes

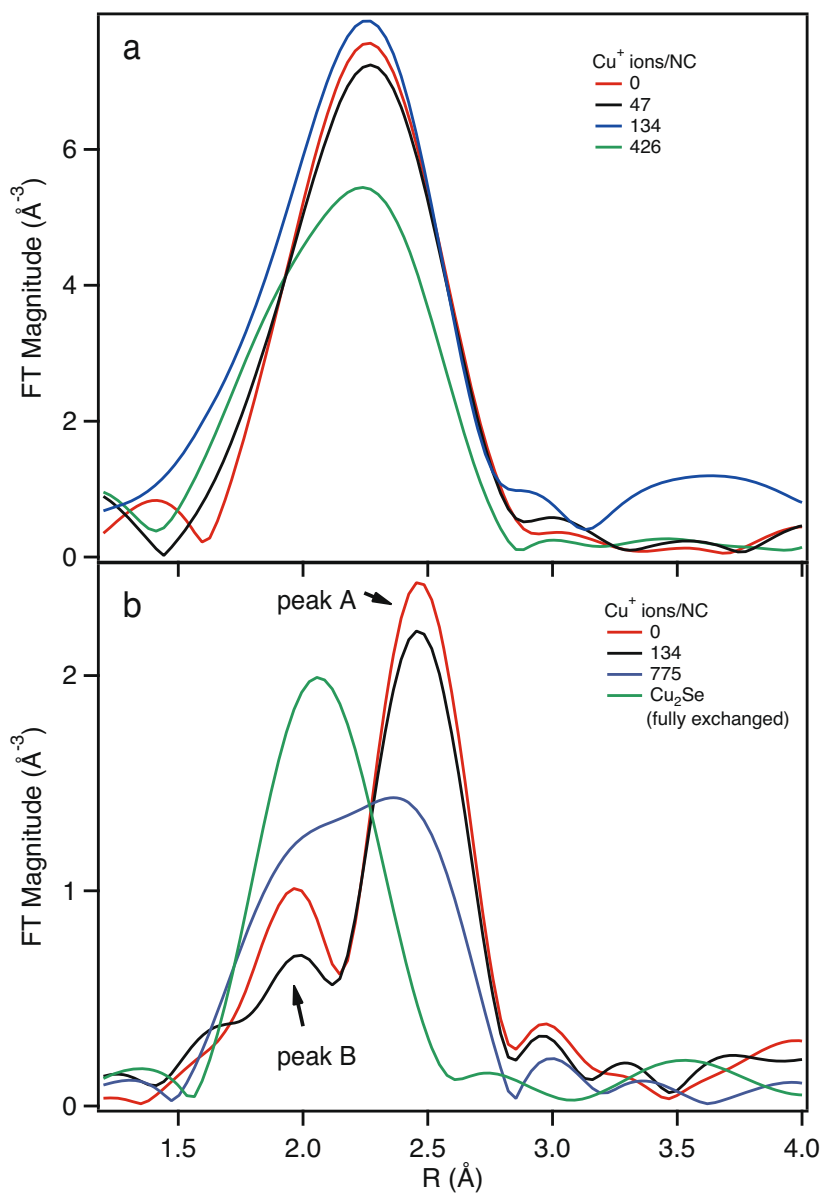

Fig. 3 Fourier transformed EXAFS data at the Cde and Se K-edges. Magnitude of the (a) Cd K-edge and (b) Se K-edge Fourier transformed EXAFS data for $5.0 \mathrm{~nm}$ CdSe NCs at various Cu concentrations. that arise during this reaction occur $\sim 1.76 \AA$ which is $\sim 0.15 \AA$ lower than the expected location of the $\mathrm{Cu}$ interstitial (not phase shift corrected) and (2) the broadening is present even when there is no observable changes in the XANES features (i.e., when the CE process has not produced any CdO-like byproducts) [see Fig. S5].

The Se K-edge FT EXAFS data can also be used for qualitative understanding of the CE process, although the interpretation is slightly more involved. Figure $3 \mathrm{~b}$ plots the Se K-edge FT EXAFS spectra for undoped $\mathrm{CdSe}, \mathrm{mol} \mathrm{Cu} / \mathrm{mol} \mathrm{Cd}$ of 0.12 and 2.9, and $\mathrm{Cu}_{2} \mathrm{Se}$ (i.e., fully exchanged). Immediately obvious is the strong overlap between the $\mathrm{NN}$ scattering peak for $\mathrm{Cu}_{2} \mathrm{Se}$, the expected scattering contribution for an interstitial $\mathrm{Cu}$ in $\mathrm{CdSe}$, and the small component of the NN scattering peak (peak A) for undoped CdSe (Fig. S6). As the $\mathrm{Cu}_{2} \mathrm{Se} \mathrm{NN}$ scattering peak occurs $\sim 0.1 \AA$ higher than undoped CdSe peak $\mathrm{A}$ and the $\mathrm{Cu}$ interstitial peak is $\sim 0.1 \AA$ lower relative to the same peak, we postulate that monitoring changes in the amplitude of the CdSe peak A can provide insight into the $\mathrm{CE}$ process. For instance, we would expect that the introduction of the $\mathrm{Cu}$ salt into the CdSe solution would lead to a broadening of peak $\mathrm{A}$ for interstitial $\mathrm{Cu}$ and simply a reduction in intensity for substitutional $\mathrm{Cu}$. As more $\mathrm{Cu}$ is introduced and the $\mathrm{CE}$ proceeds and eventually promotes the conversion of $\mathrm{CdSe}$ to $\mathrm{Cu}_{2} \mathrm{Se}$, we would expect an increase in amplitude and a shifting of peak $\mathrm{A}$ to lower $R$ towards the expected position for the main $\mathrm{NN}$ scattering peak in $\mathrm{Cu}_{2} \mathrm{Se}$. When inspecting Fig. 3, the expected behavior is observed. For lower concentrations of $\mathrm{Cu}(\mathrm{mol} \mathrm{Cu} / \mathrm{mol} \mathrm{Cd}=0.12 ; \sim 134 \mathrm{Cu}$ atoms/NC), we observe a broadening of peak $\mathrm{A}$ which is consistent with interstitial $\mathrm{Cu}$ and the conclusions from the $\mathrm{Cd}$ K-edge EXAFS results. For large concentrations of $\mathrm{Cu}(\mathrm{mol} \mathrm{Cu} /$ mol Cd $=2.9 ; \sim 775 \mathrm{Cu}$ atoms/NC), peak A has been replaced by a larger amplitude peak which is shifted on the order of $0.1 \AA$ and is consistent with a co-existence of both $\mathrm{Cu}_{2} \mathrm{Se}$ and $\mathrm{CdSe}$. The fully exchanged material is $100 \% \mathrm{Cu}_{2} \mathrm{Se}$ and exhibits only one scattering component, as expected.

We finally note that while it is understood that for every two interstitial $\mathrm{Cu}$ atoms incorporated into the $\mathrm{NC}$ we would require one Cd vacancy, we chose to exclude the inclusion of $\mathrm{Cd}$ vacancies in our calculations. Even with this exclusion, the qualitative correlations between our simulations and experiment lends credence to our approach and suggest a simple mechanism of $\mathrm{Cu}$ inclusion followed by $\mathrm{Cd}$ vacancy formation and transformation into $\mathrm{Cu}_{2} \mathrm{Se}$.

\section{Discussion}

Details on the CE mechanism can be ascertained quantitatively via fitting of the EXAFS data. We first fit the starting CdSe solution (i.e., with no added $\mathrm{Cu}$ ) to a wurtzite CdSe model and use the fitted amplitude factor $\left(S_{0}^{2}\right)$ as a set parameter for the remainder of the fits (i.e., with $\mathrm{Cu}$ added to the solution) to extract the coordination numbers, $\mathrm{CN}_{\mathrm{i}}$. To consider the inclusion of $\mathrm{Cu}$, we introduce a scattering path related to $\mathrm{Cu}$ interstitials $\left(\mathrm{Cu}_{\mathrm{i}}\right)$. Of course, during the course of the $\mathrm{CE}$ reaction, conversion of $\mathrm{CdSe}$ to $\mathrm{Cu}_{2} \mathrm{Se}$ leads to the formation of a $\mathrm{Cd}^{2+}$ byproduct, which we model in terms of a CdO scattering path for the Cd Kedge EXAFS fitting. For the Se K-edge EXAFS fitting, we include a scattering path related to $\mathrm{Cu}-\mathrm{Se}$ scattering in the $\mathrm{Cu}_{2} \mathrm{Se}$ crystal, which is distinct from the $\mathrm{Cu}$ interstitial path. All of the fitting paths are treated holistically, with the rejection (inclusion) of a path during a fit resulting from unphysical fit values (an improvement of the R-factors from the fit). As an example, in Figure S7 we display fits of the Cd K-edge FT EXAFS data for 5.0 $\mathrm{nm} \mathrm{CdSe}$ with $426 \mathrm{Cu}^{+}$ions/NC added. In this example, both the inclusion or exclusion of $\mathrm{Cu}_{\mathrm{i}}$ lead to reasonable fits, but the inclusion of $\mathrm{Cu}_{\mathrm{i}}$ results in a $40 \%$ improvement in the fit $\mathrm{R}$-factors 

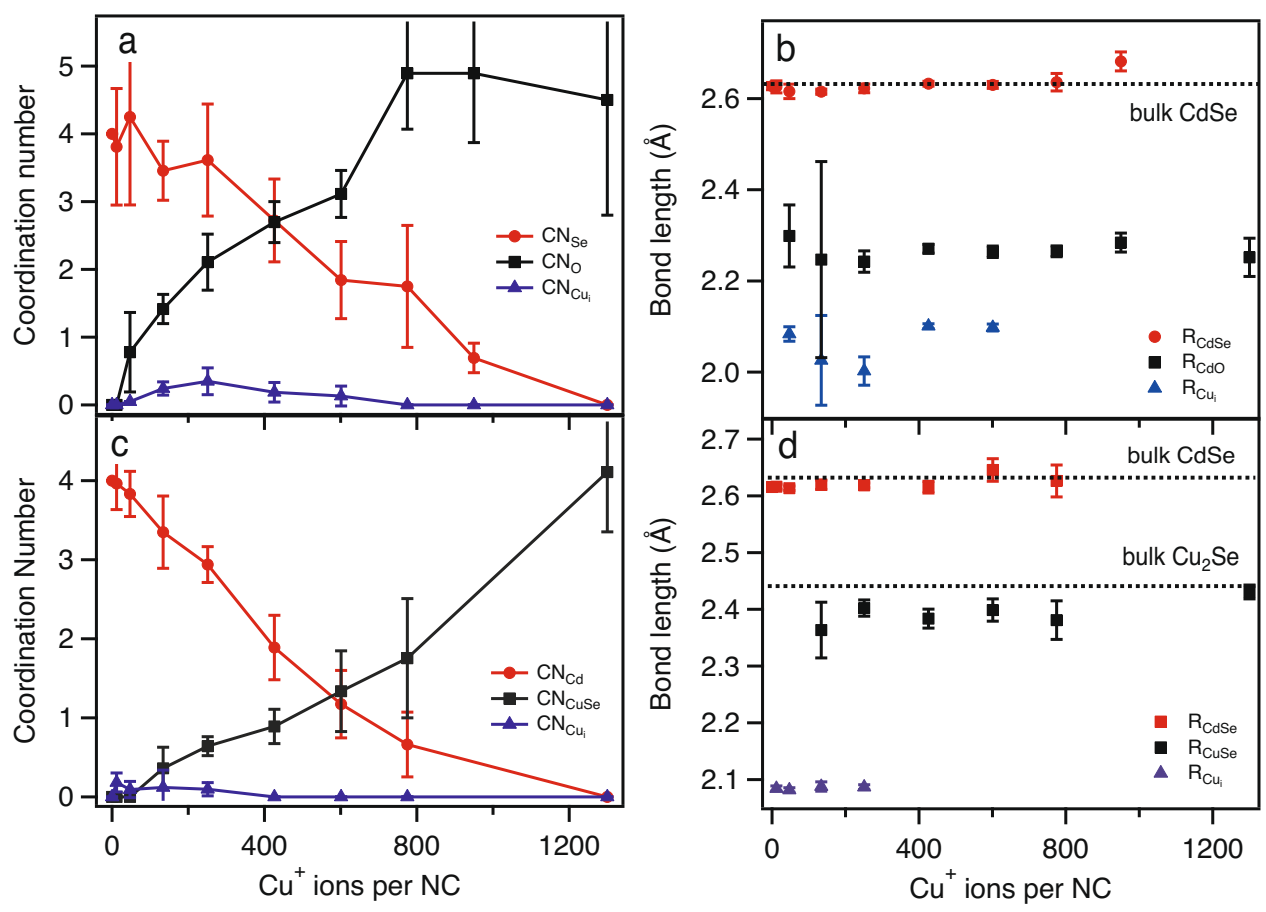

Fig. 4 Change in coordination numbers and bond lengths as a function of copper concentration. Values of the coordination numbers and bond lengths from fits of the Cd K-edge (a, b) and Se K-edge (c, d) EXAFS data for $5.0 \mathrm{~nm}$ CdSe at various stages of the copper cation exchange reaction. The error bars represent uncertainties in the EXAFS fitting, and specific details on how these errors are calculated can be found in Ref. 33 .

(0.006 vs. 0.01$)$ which allows the statement that the interstitial path is present during that concentration step. All of the fitted EXAFS data can be found in the SI. For ease of the discussion, we will focus on the $5.0 \mathrm{~nm}$ CdSe NC, as we have done throughout the entire text.

Figure 4 displays the coordination numbers, $\mathrm{CN}_{\mathrm{i}}$, and bond lengths, $R_{\mathrm{i}}$, derived from the EXAFS fits for $5.0 \mathrm{~nm}$ CdSe NCs. The general behavior for the coordination numbers for the Cd Kedge (Fig. 4a) and the Se K-edge (Fig. 4c) is similar. With increasing $\mathrm{Cu}$ concentration, the average values for the coordination numbers for Se $(\mathrm{Cd})$ for the $\mathrm{Cd}$ (Se) K-edge decrease and eventually are zero at a critical $\mathrm{Cu}$ concentration, as expected for complete conversion from $\mathrm{CdSe} \rightarrow \mathrm{Cu}_{2} \mathrm{Se}$. Conversely (but expectedly), the average values of the coordination numbers for $\mathrm{O}$ $(\mathrm{Cu})$ for the $\mathrm{Cd}(\mathrm{Se}) \mathrm{K}$-edge increase with increasing $\mathrm{Cu}$ concentration. The coordination number for the $\mathrm{Cu}_{\mathrm{i}}$ path increases with $\mathrm{Cu}$ concentration, reaches a maximum, and then decreases towards zero with increasing $\mathrm{Cu}$ concentration. Values for $\mathrm{CN}_{\mathrm{O}}$ tend to asymptote near 5-6, which suggest some form of a five- or six-fold coordinate $\mathrm{Cd}^{2+}$ complex. We note that we cannot exactly quantify the state of the $\mathrm{Cd}$ byproduct but the derived bond lengths $\left(R_{\mathrm{CdO}} \sim 2.3 \AA\right)$ support the existence of a five- or six-fold coordinate $C d$ species bonded to a low- $Z$ element ${ }^{24}$. The values for $R_{\mathrm{CdSe}}$ and $R_{\mathrm{CuSe}}$ are both remarkably consistent throughout the concentration steps, again supporting the observation of a two phase process, as severe structural perturbations or the creation of new phases could lead to much larger deviations in the derived bond lengths ${ }^{25}$. In addition, the fitted values of $R_{\mathrm{Cu}_{\mathrm{i}}} \sim 2.1 \AA$ which are similar values to $R_{\mathrm{Cu}_{\mathrm{i}}}$ that have been observed in related materials ${ }^{26}$.

While the critical variable which controls the degree of complete cation exchange is the copper concentration, this is fundamentally an extensive property that has no transferability to another system (i.e., different sized particles). The number of copper atoms present per total atoms present in the $\mathrm{NC}\left(\frac{N_{\mathrm{Cu}}}{N_{\mathrm{T}}}\right)$, however, should represent an intensive property that should represent a meaningful and comparable metric to compare across various length scales. Figure 5 a displays the values of $\mathrm{CN}$ as a function of $\frac{N_{\mathrm{Cu}}}{N_{\mathrm{T}}}$ derived from the fits of the Cd K-edge EXAFS data for three different particle sizes. Interestingly, when plotted against this atom normalized variable $\left(\frac{N_{\mathrm{Cu}}}{N_{\mathrm{T}}}\right)$, the size-dependent behavior is remarkably similar. The plots for $\mathrm{CN}_{\mathrm{Se}}$ and $\mathrm{CN}_{\mathrm{O}}$ intersect at $\frac{N_{\mathrm{Cu}}}{N_{\mathrm{T}}} \sim 0.2$ irrespective of particle size. In addition, all sizes show an increase and subsequent decrease in $\mathrm{CN}_{\mathrm{Cu}_{\mathrm{i}}}$ as a function of $\frac{N_{\mathrm{Cu}}}{N_{\mathrm{T}}}$.

This size-independent behavior in $\mathrm{CN}$ with $\mathrm{Cu}$ concentration speaks to a general phenomenon across length scales. We also point out that this type of behavior is consistent with observations by other groups using different probing methods. For instance, the Jain group used optical spectroscopy ${ }^{7}$ to probe the CE process of $\mathrm{Cu}$ in $\mathrm{CdSe}$ and found a similar crossover behavior but at a different critical concentration than we report $\left(\frac{N_{\mathrm{Cu}}}{N_{\mathrm{T}}} \sim 0.5\right)$. We note, however, that the $y$-axis of their data is in terms of the percent of NCs that have transformed from one species to another. In their data, the crossover occurs at the point where $50 \%$ of the species have converted from CdSe to $\mathrm{Cu}_{2} \mathrm{Se}$. When defined in terms of an expected $\mathrm{CN}_{\mathrm{Se}}=2$ from a $50 \%$ mixture of $\mathrm{CdSe}$ and $\mathrm{Cu}_{2} \mathrm{Se}$, the expected $\frac{N_{\mathrm{Cu}}}{N_{\mathrm{T}}} \sim 0.17$, which matches the values we report. So, it must be noted that the exact crossover point can change depending on the specific variables being observed. As an example, we also plot $\frac{N_{\mathrm{Cu}}}{N_{\mathrm{T}}}$ derived from the fits of the Se K-edge EXAFS data in Fig. 5a. For the Se K-edge data for the $5.0 \mathrm{~nm}$ CdSe NC, the crossover point occurs $\frac{N_{\mathrm{Cu}}}{N_{\mathrm{T}}} \sim 0.25$. We note that we only have high quality Se K-edge EXAFS data for the $5.0 \mathrm{~nm}$ CdSe NCs. The ability to obtain high quality data rests on a number of variables including chemical stability (precipitation during the reaction) and absorption issues (lower $Z$ elements buried in a matrix of higher $Z$ elements). 

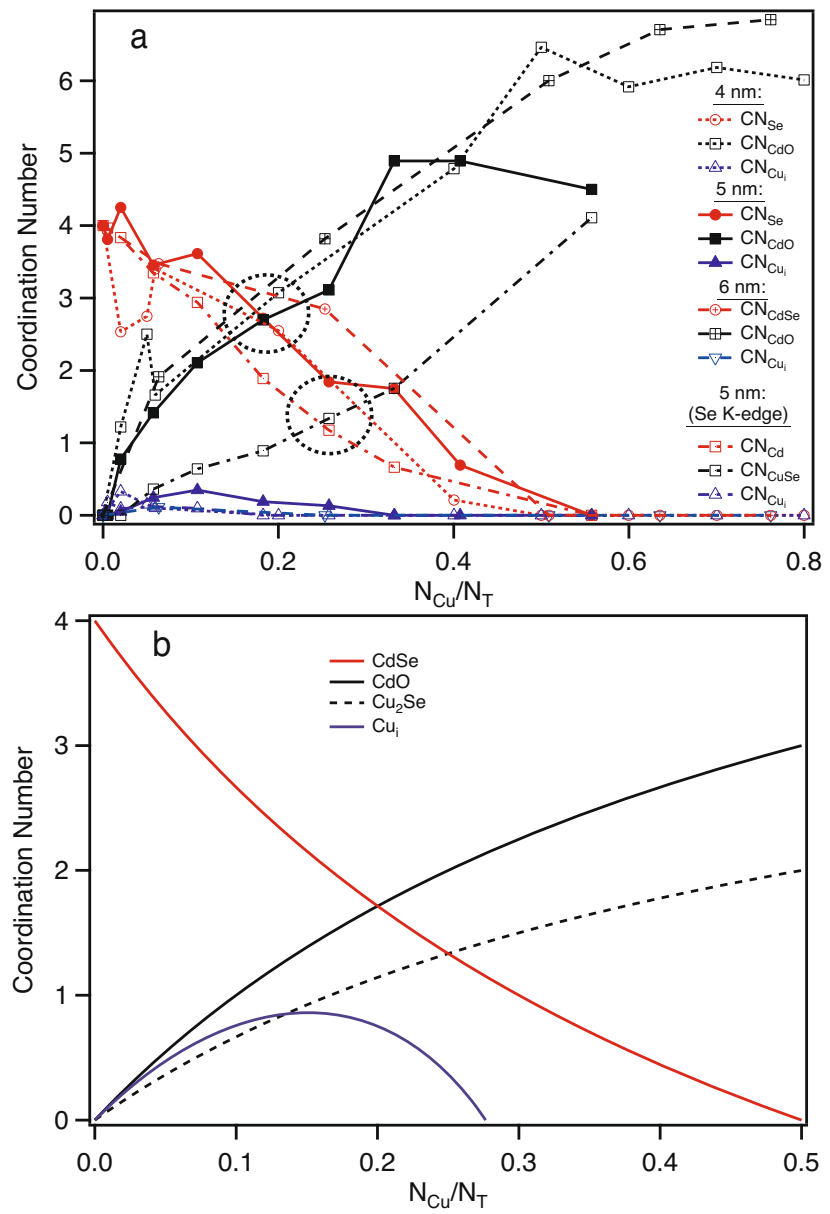

Fig. 5 Comparison of the experimental and theoretical changes in coordination number with copper concentration. Coordination number, $\mathrm{CN}$, versus the number of copper atoms $\left(\mathrm{N}_{\mathrm{Cu}}\right)$ normalized to the number of atoms $\left(\mathrm{N}_{\mathrm{T}}\right)$ in the nanocrystal. (a) Experimental data ( $\mathrm{CN}$ extracted via fits of the EXAFS data) for three different particles sizes and two different metal K-edges (error bars have been removed for clarity; for an idea of the general error associated with the data points, see the error bars in Fig. 4a and c) and (b) Theoretically predicted change in $\mathrm{CN}$ with concentration. Details on the theoretical calculations are provided in the main text.

The experimental data can be simply explained by considering the changes in the average coordination numbers as increased amounts of copper are introduced during the stages of the CE reaction. For comparison, we have calculated and plotted (Fig. 5b) the expected change in coordination number, $\mathrm{CN}$, as a function of $\mathrm{Cu}$ concentration $\left(\frac{N_{\mathrm{Cu}}}{N_{\mathrm{T}}}\right)$ based upon the following relationships (the justification for these equations can be found in Methods),

$$
\begin{gathered}
\langle\mathrm{CN}\rangle_{\mathrm{CdSe}}=\frac{2-4 \frac{N_{\mathrm{Cu}}}{N_{\mathrm{T}}}}{0.5+\frac{N_{\mathrm{Cu}}}{N_{\mathrm{T}}}} ;\langle\mathrm{CN}\rangle_{\mathrm{CdO}}=\frac{6 \frac{N_{\mathrm{Cu}}}{N_{\mathrm{T}}}}{0.5+\frac{N_{\mathrm{Cu}}}{N_{\mathrm{T}}}} \\
\langle\mathrm{CN}\rangle_{\mathrm{CuSe}}=\frac{4 \frac{N_{\mathrm{Cu}}}{N_{\mathrm{T}}}}{0.5+\frac{N_{\mathrm{Cu}}}{N_{\mathrm{T}}}} ;\langle\mathrm{CN}\rangle_{\mathrm{Cu}_{\mathrm{i}}}=\frac{8 \frac{N_{\mathrm{Cu}}}{N_{\mathrm{T}}}}{0.5+\frac{N_{\mathrm{Cu}}}{N_{\mathrm{T}}}}+\frac{\mathrm{CN}_{\mathrm{i}} \frac{N_{\mathrm{Cu}}}{N_{\mathrm{T}}}}{\frac{N_{\mathrm{Cu}}}{N_{\mathrm{T}}}-0.5}
\end{gathered}
$$

where $\mathrm{CN}_{\mathrm{i}}$ is the value of the $\mathrm{CN}$ for interstitial copper at the critical concentration where $\sim 50 \%$ of the CdSe species have converted to $\mathrm{Cu}_{2} \mathrm{Se}$. The similarity in the behavior of the experimentally and predicted changes in $\mathrm{CN}$ with concentration are striking. The theoretically expected crossover point for the $\mathrm{CdSe} / \mathrm{CdO}$ species occurs at $\frac{N_{\mathrm{Cu}}}{N_{\mathrm{T}}} \sim 0.2$ while the crossover point for the $\mathrm{CdSe} / \mathrm{Cu}_{2} \mathrm{Se}$ species occurs at $\frac{N_{\mathrm{Cu}}}{N_{\mathrm{T}}} \sim 0.25$, as observed experimentally. We can also replicate the experimental behavior in the values for $\mathrm{CN}_{\mathrm{i}}$. As a whole, we have shown that the copper cation exchange process in a series of different sized CdSe nanocrystals is explained by interstitial inclusions of $\mathrm{Cu}$ at the early stages followed by conversion of $\mathrm{CdSe} \rightarrow \mathrm{Cu}_{2} \mathrm{Se}$, with the appearance of no other spurious phases.

\section{Methods}

Materials. Cadmium carbonate $\left(\mathrm{CdCO}_{3}\right)(98 \%$, Aldrich), selenium powder $(\mathrm{Se})$ (100 mesh, $99.5 \%$ trace metals basis, ACROS), Tetrakis(acetonitrile)copper(I) hexafluorophosphate (97\%, Aldrich), tri-n-octylphosphine oxide (TOPO) $(99 \%$, Aldrich), tri-n-octylphosphine (TOP) (90\%, Aldrich), 1-octadecene (ODE) $(90 \%$, Aldrich), stearic acid (SA) (95\%), toluene (99.5\%, VWR), methanol (Fisher, $99.9 \%$ ), and ethanol (Alfa Aesar, 94-96\%). All chemicals were purchased and used without further purification.

Synthesis of CdSe nanocrystals. CdSe nanocrystals (NCs) were synthesized via a modified hot injection method from Shakeri ${ }^{19}$ and Webber ${ }^{20}$. A typical synthesis involves stirring $3.4 \mathrm{mmol}(590 \mathrm{mg})$ of $\mathrm{CdCO}_{3}$ with $5.0 \mathrm{~g}$ of TOPO and $5.0 \mathrm{~g}$ of SA in a three-neck, round bottom flask, heated to $120^{\circ} \mathrm{C}$ under flowing $\mathrm{N}_{2}$. The solution is held isothermally for $1.5 \mathrm{~h}$ before heating to $360^{\circ} \mathrm{C}$. Separately, 4.9 mmol $(383 \mathrm{mg})$ of Se powder is dissolved in $5.0 \mathrm{~mL}$ of TOP under $\mathrm{N}_{2}$. The Se solution is quickly injected into the reaction flask and followed by immediate injection of $5.0 \mathrm{~mL}$ of ODE. To quench the reaction, the flask is removed $2-5 \mathrm{~min}$ after the first injection or once the desired size is achieved. When the NC solution approaches $50^{\circ} \mathrm{C}, 5 \mathrm{~mL}$ of toluene is injected to facilitate the purification process. Once at room temperature, the mixture is distributed into three $45-\mathrm{mL}$ centrifuge tubes. Each centrifuge tube receives $5 \mathrm{~mL}$ of toluene, $6 \mathrm{~mL}$ of ethanol, and $5 \mathrm{~mL}$ of methanol. The mixture is shaken for $30 \mathrm{~s}$ and centrifuged at $3050 \mathrm{rpm}$ for $5 \mathrm{~min}$. The supernatant is decanted and the precipitate is purified twice. The purification process is continued by re-dispersing the NCs in $5 \mathrm{~mL}$ of toluene and adding $6 \mathrm{~mL}$ of ethanol and $5 \mathrm{~mL}$ of methanol to each centrifuge tube. The tubes are then shaken for $30 \mathrm{~s}$ and centrifuged for $5 \mathrm{~min}$. The final product was dried under flowing $\mathrm{N}_{2}$ overnight and sealed under a $\mathrm{N}_{2}$ atmosphere.

\section{Copper cation exchange and X-ray absorption fine structure spectroscopy}

(XAFS). X-ray absorption fine structure (XAFS) experiments were performed at Sector 10 on the insertion device line operated by the Materials Research Collaborative Access Team (MRCAT) ${ }^{27}$. Measurements were performed in both transmission and fluorescence using a Lytle detector. XAFS measurements were performed on a $0.2 \mathrm{M}\left(5.2 \times 10^{-4} \mathrm{~mol}\right)$ toluene solvated CdSe NC solution in a custom-built peristaltic pump-driven flow cell. Aliquots of a $0.1 \mathrm{M}$ (Copper precursor) in ethanol $\left(1.1 \times 10^{-3} \mathrm{~mol}\right)$ were added to the stirred CdSe solution and allowed to equilibrate. The concentration steps are presented for completeness in the Supporting Information (Table S1). After addition of the copper salt solution, XAFS scans were taken at the $\mathrm{Cu}, \mathrm{Cd}$, and Se K-edges. Calibrations were done on $\mathrm{Cd}\left(\mathrm{NO}_{3}\right)_{2}$ powder, or $\mathrm{Cu}$ and Se foils for the $\mathrm{Cd}, \mathrm{Cu}$, and Se edges, respectively.

XAFS data processing and analysis were done using the IFEFFIT suite of programs ${ }^{28}$. Initial estimates of the threshold energy values $\left(E_{0}\right)$ were obtained via the inflection point in the normalized absorption edges. A Hanning window was applied to a selected $k$-range (nominally $3-11 \AA^{-1}$ ) to obtain the Fourier transformed (FT) extended XAFS (EXAFS) data. For quantitative determination of both coordination numbers $\left(\mathrm{CN}_{i}\right)$, bond lengths $\left(R_{i}\right)$, and EXAFS Debye-Waller factors (static disorders, $\sigma_{i}^{2}$ ), the experimental data was fit using $\mathrm{CdSe}, \mathrm{Cu}_{2} \mathrm{Se}$, interstitial $\mathrm{Cu}$, or $\mathrm{CdO}$ as the fitting models. FEFF6 was used to calculate the photoelectron scattering path amplitudes, $F_{i}(k)$, and phase, $\phi(k)$, and the samples were fit to the EXAFS equation,

$$
\chi(k)=\frac{\left(C N_{i} S_{o}^{2}\right)}{\left(2 k R_{i}^{2}\right)} F_{i}(k) e^{\left(-2 k^{2} \sigma_{i}^{2}\right)} \sin \left[2 k R_{i}+\phi_{i}(k)\right]
$$

Ultra-violet Visible (UV-Vis) and Photoluminescence (PL) Spectroscopy. The $\mathrm{UV}-\mathrm{V}$ is spectra were recorded in toluene on an Ocean Optics

USB2000 spectrophotometer using a $1 \mathrm{~cm}$ path length quartz cell. The absorbance values of the first absorption maximum were used to calculate the NC diameters using the empirical formula reported by Peng and co-workers ${ }^{29}$ and Mulvaney and co-workers ${ }^{30}$. PL spectra were measured using an excitation wavelength of $\lambda_{\text {max }}=$ $405 \mathrm{~nm}(E=3.06 \mathrm{eV})$ and the signal collected using an Ocean Optics USB2000 CCD spectrometer.

X-ray Diffraction (XRD). NC structure was studied using XRD by $\theta-2 \theta$ scans with $\mathrm{Cu} \mathrm{K} \alpha$ radiation using line focus on a PANalytical X'PertPro Diffractometer. XRD samples were prepared by drop-casting a small amount of the NC solution onto a glass slide. 
Theoretical details. To calculate the CdSe coordination number, $\langle\mathrm{CN}\rangle_{\mathrm{CdSe}}$, we calculate,

$$
\langle\mathrm{CN}\rangle_{\mathrm{CdSe}}=\frac{\mathrm{CN}_{\text {bulk }}^{\mathrm{CdSe}}(n-m)}{n+m}
$$

where $n=\#$ of $\mathrm{Cd}$ atoms and $m=\#$ of $\mathrm{Cu}$ atoms. We re-define $m \equiv \mathrm{N}_{\mathrm{Cu}}$ and $n \equiv$ $\mathrm{N}_{\mathrm{Cd}}$ and note that $\mathrm{N}_{\mathrm{Cd}}=\mathrm{N}_{\mathrm{T}} / 2$, where $\mathrm{N}_{\mathrm{T}}$ is the total \# of atoms in the undoped NC. We realize that this is a crude assumption on the exact stoichiometry of CdSe $\mathrm{NCs}^{31,32}$, but for our purposes, we find it an appropriate approximation. The above expression then becomes,

$$
\langle\mathrm{CN}\rangle_{\mathrm{CdSe}}=\frac{\mathrm{CN}_{\text {bulk }}^{\mathrm{CdSe}}\left(N_{\mathrm{T}} / 2-N_{\mathrm{Cu}}\right)}{N_{\mathrm{T}} / 2+N_{\mathrm{Cu}}}
$$

We divide through by $N_{\mathrm{T}}$ and note that $\mathrm{CN}_{\text {bulk }}^{\text {CdSe }}=4$, we can write,

$$
\langle\mathrm{CN}\rangle_{\mathrm{CdSe}}=\frac{2-4 N_{\mathrm{Cu}} / N_{\mathrm{T}}}{0.5+N_{\mathrm{Cu}} / N_{\mathrm{T}}}
$$

For the $\mathrm{CdO}$ or $\mathrm{Cu}_{2} \mathrm{Se}$ coordination environments, we follow similar logic but only account for the number of $\mathrm{CdO}$ or $\mathrm{Cu}_{2} \mathrm{Se}$ that is created,

$$
\langle\mathrm{CN}\rangle_{\mathrm{CdO} / \mathrm{CuSe}}=\frac{\mathrm{CN}_{\mathrm{bulk}}^{\mathrm{CdO} / \mathrm{CuSe}} m}{n+m}
$$

Using $\mathrm{CN}_{\text {bulk }}^{\mathrm{CdO}}=6$ and $\mathrm{CN}_{\text {bulk }}^{\mathrm{CuSe}}=4$, we arrive at,

$$
\begin{aligned}
& \langle\mathrm{CN}\rangle_{\mathrm{CdO}}=\frac{6 \frac{N_{\mathrm{Cu}}}{N_{\mathrm{T}}}}{0.5+\frac{N_{\mathrm{Cu}}}{N_{\mathrm{T}}}} \\
& \langle\mathrm{CN}\rangle_{\mathrm{CuSe}}=\frac{4 \frac{N_{\mathrm{Cu}}}{N_{\mathrm{T}}}}{0.5+\frac{N_{\mathrm{Cu}}}{N_{\mathrm{T}}}}
\end{aligned}
$$

To derive the equation for interstitial $\mathrm{Cu}$, we note that we expect the number to $\mathrm{Cu}$ interstitials to increase with concentration up to a critical point denote by some coordination number $\mathrm{CN}_{\mathrm{i}}$, at which there is enough $\mathrm{Cu}$ present to form $\mathrm{Cu}_{2} \mathrm{Se}$ and therefore results in a decrease in $\mathrm{Cu}$ interstitial concentration. We can expect, then, that such a dependence can be written as,

$$
\begin{gathered}
\langle\mathrm{CN}\rangle_{\mathrm{Cu}_{\mathrm{i}}}=\frac{\mathrm{CN}_{\text {bulk }}^{\mathrm{i}} m}{n+m}+\frac{\mathrm{CN}_{\mathrm{i}} m}{n+m-2 m} \\
\langle\mathrm{CN}\rangle_{\mathrm{Cu}_{\mathrm{i}}}=\frac{\mathrm{CN}_{\text {bulk }}^{\mathrm{i}} m}{n+m}+\frac{\mathrm{CN}_{\mathrm{i}} m}{n-m}
\end{gathered}
$$

We note that the term $(-2 m)$ appears in the denominator because due to the stoichiometry of the CE reaction. Using similar logic as above, we can re-write this expression as,

$$
\langle\mathrm{CN}\rangle_{\mathrm{Cu}_{\mathrm{i}}}=\frac{8 \frac{N_{\mathrm{Cu}}}{N_{\mathrm{T}}}}{0.5+\frac{N_{\mathrm{Cu}}}{N_{\mathrm{T}}}}+\frac{\mathrm{CN}_{\mathrm{i}} \frac{N_{\mathrm{Cu}}}{N_{\mathrm{T}}}}{\frac{N_{\mathrm{Cu}}}{N_{\mathrm{T}}}-0.5}
$$

where $\mathrm{CN}_{\mathrm{i}}$ is the value of the $\mathrm{CN}$ for interstitial copper at the critical concentration where $\sim 50 \%$ of the CdSe species have converted to $\mathrm{Cu}_{2} \mathrm{Se}$.

\section{Data availability}

The data that support the findings of this study are available from the corresponding author upon reasonable request.

Received: 10 June 2020; Accepted: 11 December 2020; Published online: 19 January 2021

\section{References}

1. Murray, C. B., Norris, D. J. \& Bawendi, M. G. Synthesis and characterization of nearly monodisperse $\mathrm{CdE}(\mathrm{E}=\mathrm{S}$, Se, Te) semiconductor nanocrystallites. $J$. Am. Chem. Soc. 115, 8706-8715 (1993).

2. Pradhan, N., Goorskey, D., Thessing, J. \& Peng, X. G. An alternative of CdSe nanocrystal emitters: pure and tunable impurity emissions in $\mathrm{ZnSe}$ nanocrystals. J. Am. Chem. Soc. 127, 17586-17587 (2005).

3. Ma, C., Moore, D., Li, J. \& Wang, Z. L. Nanobelts, nanocombs, and nanowindmills of Wurtzite ZnS. Adv. Mater. 15, 228 (2003).

4. Yu, W. W. \& Peng, X. G. Formation of high-quality CdS and Other II-VI semiconductor nanocrystals in noncoordinating solvents: tunable reactivity of monomers. Angew. Chem. Int. Ed. 41, 2368-2371 (2002).

5. Park, J., Joo, J., Kwon, S. G., Jang, Y. \& Hyeon, T. Synthesis of monodisperse spherical nanocrystals. Angew. Chem. Int. Ed. 46, 4630-4660 (2007).

6. Son, D. H., Hughes, S. M., Yin, Y. D. \& Alivisatos, A. P. Cation exchange reactions in ionic nanocrystals. Science 306, 1009-1012 (2004).
7. White, S. L., Smith, J. G., Behl, M. \& Jain, P. K. Co-operativity in a nanocrystalline solid-state transition. Nature Comm. 4, 2933 (2013).

8. Li, H. B. et al. Sequential cation exchange in nanocrystals: preservation of crystal phase and formation of metastable phases. Nano Lett. 11, 4964-4970 (2011).

9. Ott, F.D., Spiegel, L.L., Norris, D.J., Erwin, S.C. Microscopic theory of cation exchange in Cdse nanocrystals. Phys. Rev. Lett.113, 156803 (2014).

10. Lesnyak, V., Brescia, R., Messina, G. C. \& Manna, L. Cu vacancies boost cation exchange reactions in copper selenide nanocrystals. J. Am. Chem. Soc. 137, 9315-9323 (2015).

11. Beberwyck, B. J., Surendranath, Y. \& Alivisatos, A. P. Cation exchange: a versatile tool for nanomaterials synthesis. J. Phys. Chem. C 117, 19759-19770 (2013).

12. Koscher, B. A., Bronstein, N. D., Olshansky, J. H., Bekenstein, Y. \& Alivisatos, A. P. Surface- vs diffusion-limited mechanisms of anion exchange in $\mathrm{CsPbBr} 3$ Nanocrystal Cubes Revealed through Kinetic Studies. J. Am. Chem. Soc. 138, 12065-12068 (2016).

13. Fan, Z., Lin, L.-C., Buijs, W., Vlugt, T. J. H. \& van Huis, M. A. Atomistic understanding of cation exchange in $\mathrm{PbS}$ nanocrystals using simulations with pseudoligands. Nature Comm. 7, 11503 (2016).

14. Anpo, M., Yamashita, H., Ichihashi, Y., Fujii, Y. \& Honda, M. Photocatalytic reduction of $\mathrm{CO}_{2}$ with $\mathrm{H}_{2} \mathrm{O}$ on titanium oxides anchored within micropores of zeolites: effects of the structure of the active sites and the addition of Pt. J. Phys. Chem. B 101, 2632-2636 (1997)

15. Gunter, M. M., Ressler, T., Jentoft, R. E. \& Bems, B. Redox behavior of copper oxide/zinc oxide catalysts in the steam reforming of methanol studied by in situ X-ray diffraction and absorption spectroscopy. J. Catal. 203, 133-149 (2001).

16. Tinnemans, S. J. et al. Combining operando techniques in one spectroscopicreaction cell: new opportunities for elucidating the active site and related reaction mechanism in catalysis. Catal. Today 113, 3-15 (2006).

17. Newman, C. et al. Effects of support identity and metal dispersion in supported ruthenium hydrodeoxygenation catalysts. Appl. Catal. A 477, 64-74 (2014).

18. Kompch, A. et al. Localization of Ag dopant atoms in CdSe nanocrystals by reverse Monte Carlo analysis of EXAFS spectra. J. Phys. Chem. C 119, 18762-18772 (2015).

19. Shakeri, B. \& Meulenberg, R. W. A closer look into the traditional purification process of CdSe semiconductor quantum dots. Langmuir 31, 13433-13440 (2015).

20. Webber, D. H. \& Brutchey, R. L. Ligand exchange on colloidal CdSe nanocrystals using thermally labile tert-Butylthiol for improved photocurrent in nanocrystal films. J. Am. Chem. Soc. 134, 1085-1092 (2012).

21. Chan, E. M. et al. Millisecond kinetics of nanocrystal cation exchange using microfluidic X-ray absorption spectroscopy. J. Phys. Chem. A 111, 12210-12215 (2007).

22. Sharma, M. et al. Understanding the journey of dopant copper ions in atomically flat colloidal nanocrystals of CdSe nanoplatelets using partial cation exchange reactions. Chem. Mater. 30, 3265-3275 (2018).

23. Rehr, J. J., Kas, J. J., Vila, F. D., Prange, M. P. \& Jorissen, K. Parameter-free calculations of x-ray spectra with FEFF9. Phys. Chem. Chem. Phys. 12, 5503-5513 (2010).

24. Chakrabarti, N., Ruccolo, S. \& Parkin, G. Cadmium compounds with an $\left[\mathrm{N}_{3}\right.$ C] atrane motif: evidence for the generation of a cadmium hydride species. Inorg. Chem. 55, 12105-12109 (2016).

25. Schmitt, R. et al. A review of defect structure and chemistry in ceria and its solid solutions. Chem. Soc. Rev. 49, 554-592 (2020).

26. Soo, Y. L. et al. Migration of constituent atoms and interface morphology in a heterojunction between CdS and single crystals. J. Appl. Phys. 86, 6052 (1999).

27. Segre, C. U. et al. The MRCAT insertion device beamline at the advanced photon source. AIP Conf. Proc. 521, 419-422 (2000).

28. Ravel, B. \& Newville, M. ATHENA, ARTEMIS, HEPHAESTUS: data analysis for X-ray absorption spectroscopy using IFEFFIT. J. Synchrotron Rad. 12, 537-541 (2005)

29. Yu, W. W., Qu, L. H., Guo, W. Z. \& Peng, X. G. Experimental determination of the extinction coefficient of CdTe, CdSe, and CdS nanocrystals. Chem. Mater. 15, 2854-2860 (2003).

30. Jasieniak, J., Smith, L., van Embden, J., Mulvaney, P. \& Califano, M. Re examination of the size-dependent absorption properties of CdSe quantum dots. J. Phys. Chem. C 113, 19468-19474 (2009).

31. Taylor, J., Kippeny, T. \& Rosenthal, S. J. Surface stoichiometry of CdSe nanocrystals determined by Rutherford Backscattering spectroscopy. J. Clust. Sci. 12, 571-582 (2001).

32. Jasieniak, J. \& Mulvaney, P. From Cd-Rich to Se-Rich - the manipulation of CdSe nanocrystal surface stoichiometry. J. Am. Chem. Soc. 129, 2841-2848 (2007).

33. Newville, M., Boyanov, B. I. \& Sayers, D. E. Estimation of uncertainties in XAFS data. J. Synchrotron Rad. 6, 264-265 (1999). 


\section{Acknowledgements}

This work was supported by the National Science Foundation under Grant No. DMR1708617. MRCAT operations are supported by the Department of Energy (DOE) and the MRCAT member institutions. This research used resources of the Advanced Photon Source, a U.S. DOE Office of Science User Facility operated for the DOE Office of Science by Argonne National Laboratory under Contract No. DE-AC02-06CH11357.

\section{Author contributions}

A.K. prepared samples and performed measurements. J.T.W. designed and performed measurements. R.W.M. conceived the project, designed and performed measurements, developed concepts and theory, and analyzed the data. The manuscript was prepared R. W.M. with input from all authors.

\section{Competing interests}

The authors declare no competing interests.

\section{Additional information}

Supplementary information is available for this paper at https://doi.org/10.1038/s41467020-20712-0.

Correspondence and requests for materials should be addressed to R.W.M.
Peer review information Nature Communications thanks the anonymous reviewers for their contribution to the peer review of this work. Peer reviewer reports are available.

Reprints and permission information is available at http://www.nature.com/reprints

Publisher's note Springer Nature remains neutral with regard to jurisdictional claims in published maps and institutional affiliations.

(c) Open Access This article is licensed under a Creative Commons Attribution 4.0 International License, which permits use, sharing, adaptation, distribution and reproduction in any medium or format, as long as you give appropriate credit to the original author(s) and the source, provide a link to the Creative Commons license, and indicate if changes were made. The images or other third party material in this article are included in the article's Creative Commons license, unless indicated otherwise in a credit line to the material. If material is not included in the article's Creative Commons license and your intended use is not permitted by statutory regulation or exceeds the permitted use, you will need to obtain permission directly from the copyright holder. To view a copy of this license, visit http://creativecommons.org/ licenses/by/4.0/.

(c) The Author(s) 2021 